\title{
X-ray tomographies of a water-sensitive granular material (couscous) ex- posed to high relative humidity: an experimental study
}

\author{
Ilija Vego ${ }^{1, *}$, Alessandro Tengattini ${ }^{1}$, Edward Andò ${ }^{1}$, Nicolas Lenoir ${ }^{1}$, and Gioacchino Viggiani ${ }^{1}$ \\ ${ }^{1}$ Univ. Grenoble Alpes, CNRS, Grenoble INP, 3SR, 38000 Grenoble, France
}

\begin{abstract}
Several granular materials are hydro-sensitive, i.e., contact with water severely affects their morphology and mechanical behaviour. To this broad class belong a series of materials of great relevance for the pharmaceutical and food industry. Former studies have been conducted on the effect that moisture or humidity have on granular flow or on the individual grain mechanical response, but the processes occurring at the microlevel and their influence on the overall granular packing behaviour is yet to be fully understood. This study presents an experimental investigation of the response of a water-sensitive material (couscous) exposed to high relative humidity (97\%). 4D (3D plus time) x-ray tomographies were acquired in operando. A data treatment approach based on Dicrete Digital Image Correlation (dDIC) is developed and detailed here. This allows for following each individual grain throughout the 4+ days of the humidification test. This, in turn, allows the study of the inter- and intra-granular strain, which is particularly pronounced in water sensitive materials. The expansion of the individual grains also severely affects the contact network, whose evolving properties are here analysed and correlated to the macroscopic (sample-scale) deformation.
\end{abstract}

\section{Introduction}

Several engineering problems originate from the complex chemo-hydro-mechanical interactions of granular media. In food and pharmaceutical industries, most materials (e.g., rice, pasta, sugar, seeds, ...) are highly affected by humidity and/or moisture. The phenomena that occur when these granular media interact with water have direct consequences on industrial processes. The product loses its quality and might be wasted, rising economical and most importantly ethical issues.

While the role of moisture on the mechanical response of impermeable granular media is relatively well understood, or at least thoroughly studied (e.g., the so-called apparent cohesion in sand [1]), organic materials have the additional complexity of the chemical interaction with water, and they are thus referred as water-sensitive, enclosing the subset of effects that has to do with water influencing the mechanics of the individual grains in terms of chemistry and swelling as opposed to the capillarity effect on the granular skeleton induced by partial saturation. Some effects of grain(s)-water interaction on mechanical properties or on flowability have been already investigated, highlighting numerous problems, such as agglomeration (bridging and caking) [2, 3], swelling [4, 5], mucilage or starch release $[6,7]$, loss of resistance [8, 9], creation of fissures [10], etc. However, these studies tend to concentrate on the behaviour of the individual grains or on the flow behaviour, leaving a dark area around what happens at the micro level or at macro-response in quasi-static conditions, which are the ones at which the product is stored (for instance silos).

*e-mail: Ilija.Vego@univ-grenoble-alpes.fr A video is available at https://doi.org/10.48448/29yv-7111
Numerical simulations could also help contain and prevent the industrial issues caused by the exposition of watersensitive materials to water, but require a proper calibration [11], which is essentially missing in this context.

This paper presents the study conducted on a watersensitive granular material: couscous. To study its hydromechanical response, a cylindrical specimen of the material is exposed for around 4 days to high relative humidity air (RH 97\%), while it is compressed under constant vertical load.

3D greyscale images are acquired continuously throughout the entire testing duration by means of x-ray tomography. These images are then analysed and measurements were made both macro (i.e., of the entire sample) and micro (of the single grain) scale. The evolution of the attenuation coefficient of the material is analysed, and the porosityvolumetric strain relation is examined. Together with these macro measurements, Discrete Digital Image Correlation (dDIC) allows following individually all $\approx 2200$ grains and measuring their deformation and kinematics. The micro and macro behaviour are then compared. Finally, the evolution of the contact area between grains is analysed.

\section{Experimental campaign}

\subsection{Sample preparation and experimental set up}

The material chosen for this experimental campaign is couscous, which is a well known for being water-sensitive. The average grain size $(0.4 \div 0.5 \mathrm{~mm}$ in diameter $)$ is compatible with the resolution of the x-ray detector of the $\mathrm{X}$-ray tomograph installed at Laboratoire 3SR. Couscous 


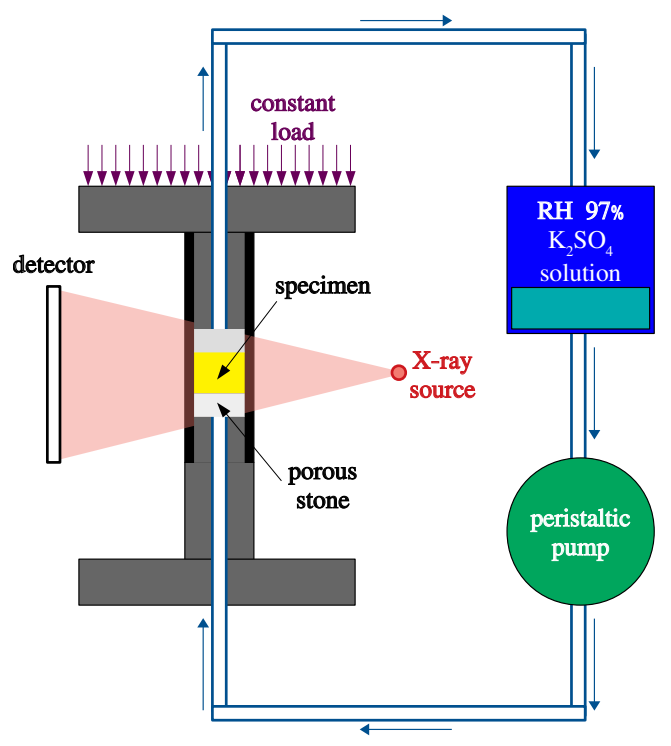

Figure 1. Experimental set-up used for this experimental campaign. Couscous grains were placed in an oedometer, under a dead load of $20 \mathrm{kPa}$. They were constantly exposed to high relative humidity air, generated in a humidity control chamber containing a potassium sulphate saturated solution $(\mathrm{RH}=97 \%)$ and pumped at $15 \mathrm{~mL} / \mathrm{min}$ with a peristaltic pump.

grains, when exposed to moisture or humidity, absorb water, increase in volume and become sticky (i.e. agglomeration/cementation). Adding further complexity, for instance choosing a material that peels off, would affect the interpretation and so the measurements of the processes occurring at the micro-level.

For the experiment, an oedometer $16 \mathrm{~mm}$ in diameter is used, with no radial strain; a constant vertical load of $20 \mathrm{kPa}$ is applied to the specimen. The cell is connected to a humidity control chamber from which high relative humidity air $(97 \%)$ flows into the oedometer $(\approx 15 \mathrm{~mL} / \mathrm{min})$ by means of a peristaltic pump. The specimen contains $1.3 \mathrm{~g}$ of dry couscous grains, and it is placed between two porous stones, to avoid the grains falling into the channels from which air enters and exits the cell.

The humidity control chamber contains $1.5 \mathrm{~L}$ of saline saturated solution, obtained with de-mineralized water $\left(\mathrm{H}_{2} \mathrm{O}\right)$ and potassium sulphate $\left(\mathrm{K}_{2} \mathrm{SO}_{4}\right)$. This solution is known to generate a $97 \%$ relative humidity environment, and it is also stable at $22 \pm 5^{\circ} \mathrm{C}[12]$.

A simple sketch of the experimental set-up is presented in Figure 1.

\subsection{Data acquisition: x-ray tomography}

$\mathrm{X}$-ray tomographies are performed continuously during the whole test, for about four days (105 hours). A tomography of the dry sample is initially acquired, after which the pump is activated, and continuous scans are collected. Each tomography lasts $1 \mathrm{~h}$, with no inter-time in between acquisition. The speed of the tomography is chosen as a compromise between the desired spatial resolution (the reached pixelsize is $14 \mu \mathrm{m}$ ) and the rate at which the phe-
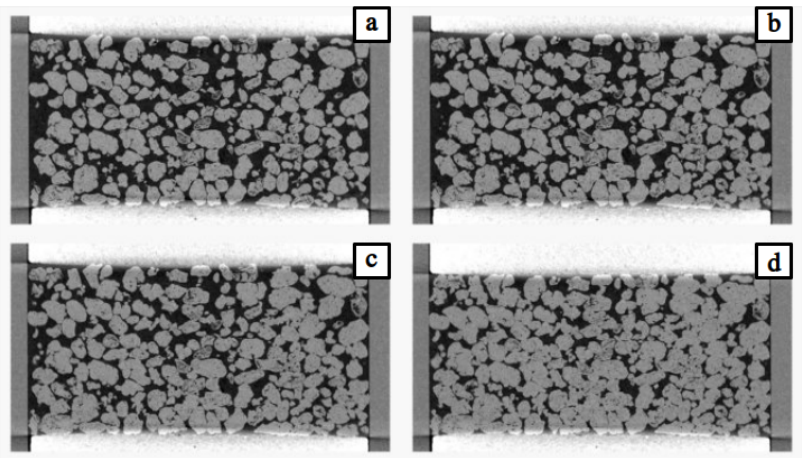

Figure 2. Vertical slices of the sample raw image data at $\mathrm{t}=0 \mathrm{~h}$ (a), $\mathrm{t}=24 \mathrm{~h}$ (b), $\mathrm{t}=48 \mathrm{~h}$ (c) and $\mathrm{t}=96 \mathrm{~h}$ (d). The sample is able to lift the deadload, but then collapses under it, while couscous grains continuously swell.

nomena occur. Each tomography comprises 1440 projections, each being the average of 7 individual acquisitions lasting $0.37 \mathrm{~s} \mathrm{each}$.

\subsection{Image analysis: results}

From the projections, a total of $1053 \mathrm{D}$ volumes are reconstructed with a Fedelkamp reconstruction algorithm with identical parameters. The overall process can be observed in Figure 2, which highlights the shortening of the sample during the test. In Figure 3 are shown the grey-values histograms of the different scans collected along the test. The histogram peak corresponding to the voids gradually decreases, which is consistent with the visible reduction of porosity. Hand in hand, the peak of the granular phase $(\approx 55000$ counts) increases, indicating a larger percentage of the sample belongs to this phase, as a consequence of grains swelling and the sample compaction, but it also shifts towards lower attenuation values, corresponding, in this case to a lower average density of the grains, compatibly with the observed attenuation. The grains exposed to humidity absorb water which is not as dense as dry couscous (the sample was indeed found to be $15 \%$ heavier at

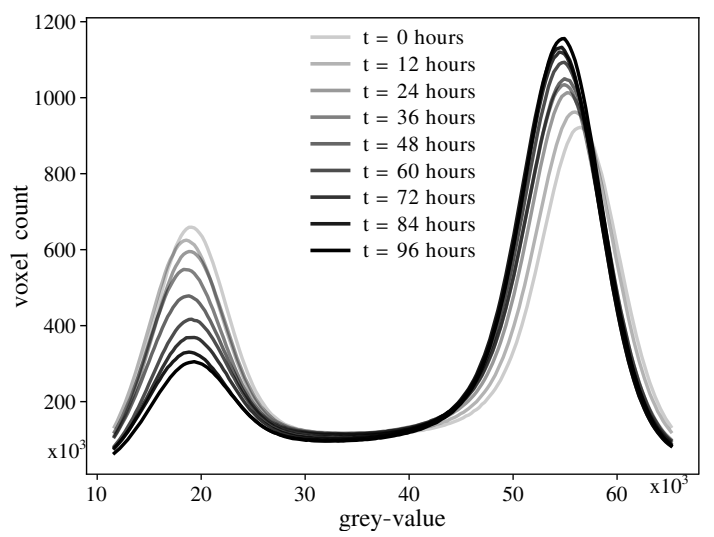

Figure 3. Grey-value histograms of the acquired images at different times during a test. 


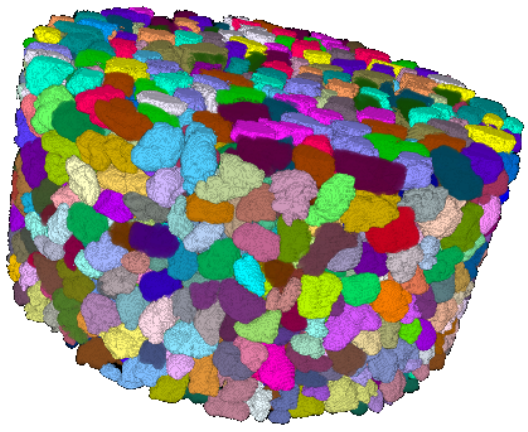

Figure 4. 3D rendering of the segmented couscous specimen. Every single grain has its own label and its kinematics can be followed thorough the process.

the end of the process). The overall reduction of the material density could be then explained as a result of the water triggering the swelling, while it is absorbed in the sub-micro pores of couscous.

To study the evolution of the porosity the voxels are classified with a fixed threshold-based segmentation, whose limit values are calculated via Otsu's method (determined by minimizing intra-class intensity variance of all the voxels), while each voxel that has a higher value, is considered to belong to the solid phase.

The overall volumetric strain of the sample is also calculated through global Digital Image Correlation and is reported in Figure 5, which shows the non-monotonic relationship with the porosity evolution detailed above. This confirms what could be intuitively observed from the reconstructed vertical slices (Figure 2). The sample is initially dilating (for about 1 day of high $\mathrm{RH}$ exposure), and only then starts compressing under the constant load applied, reaching the same size as the initial state after approximately 2 days, followed by compaction.

It can be also noticed how the evolution rate of both macro volumetric strain and porosity seem to have a strong connection. To investigate the origin of this correlation the strain of individual grains was analysed.

To follow the inter-granular strain and the evolution of the contact network it is first necessary to identify the individual grains. The first greyscale image is binarised and then segmented with an iterative watershed algorithm, in order to avoid over-segmentation caused by the highly irregular shape of couscous grains. A 3D rendering of the final segmentation result is shown in Figure 4.

Once all initial grains are identified, their evolution across the test can be followed by employing discrete Digital Image Correlation (dDIC) on each of the segmented grains. This tool, used for the first time in [13] and later implemented in the open-source software 'spam' [14], allows to measure displacement, rotation and deformation tensor of each grain. The $\mathrm{dDIC}$ algorithm, using the labelled image as a mask, iteratively deforms the sub-domains of the reference containing the grains as to find the best match with the image, while also measuring their kinematics. In this experimental campaign the step 0 (i.e. the scan obtained

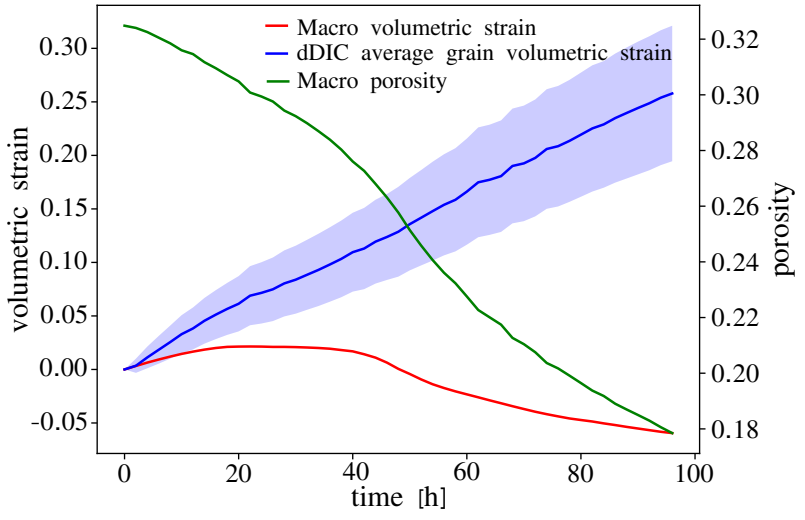

Figure 5. Comparison between the sample (red) and the average grain (blue) volumetric strains (the region represents the error margin of the grain volumetric strain) along with the sample porosity (green).

under dry condition) was used as the (only) reference image and it was possible the measurement of the kinematics and strain of all the particles, up until the end of day 4 of testing.

The dDIC returns, among other measurements (e.g., displacement), the strain tensor of each particle. Decomposing this tensor, the volumetric strain of each grain is extracted. The evolution in time of the average grain volumetric strain obtained from dDIC, along with its margin error represented by the light blue area, is shown in Figure 5, along with the macro volumetric strain and porosity. The grain volumetric strain increases through the entire exposition to high RH at basically constant rate and it is clear how the evolution of porosity is influenced by the combination of macro and micro volumetric strain. During the first hours the porosity becomes lower due to grains swelling. Later, its reduction rate increases because of the overall specimen compression. At the final steps, both macro and micro seem to be responsible of its decrease.

Once the dDIC is complete (i.e. convergence for each of the $\approx 2200$ particles), it is possible to obtain labelled images for each of the following steps. Knowing the displacements, rotations and deformation of all grains, it is indeed possible to preserve the information about the grain shape and size. This new labelled images, allow the study of the contact between the grains. Figure 6 shows the 3D rendering of two grains in contact at different times. It is evident how the contact area increases during the process, as a result of swelling, compaction and a higher deformability of grains caused by water absorption. This area is measured as the number of single voxels of the analysed grain that are "touching" the other one. The plot in Figure 7 presents the evolution in time of all the contacts of a randomly chosen particle of the assembly. All the contact areas tend to expand in time, with some exceptions in the interval of time while the specimen is increasing in volume. It can be also noticed how the number of grains in contact raises through the test. In fact, swelling and compression make the distance between particles shorter, and sometimes they generate new contacts. 


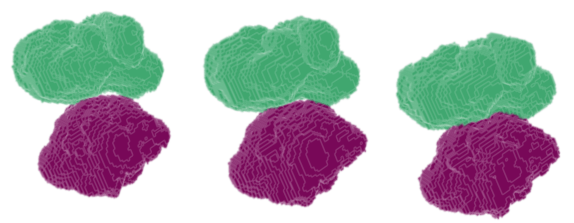

Figure 6. $3 \mathrm{D}$ rendering of two grains in contact. From left to right, $\mathrm{t}=0 \mathrm{~h}, \mathrm{t}=48 \mathrm{~h}$ and $\mathrm{t}=96 \mathrm{~h}$. The grains swelled during the high RH exposition, and so they increased their contact area.

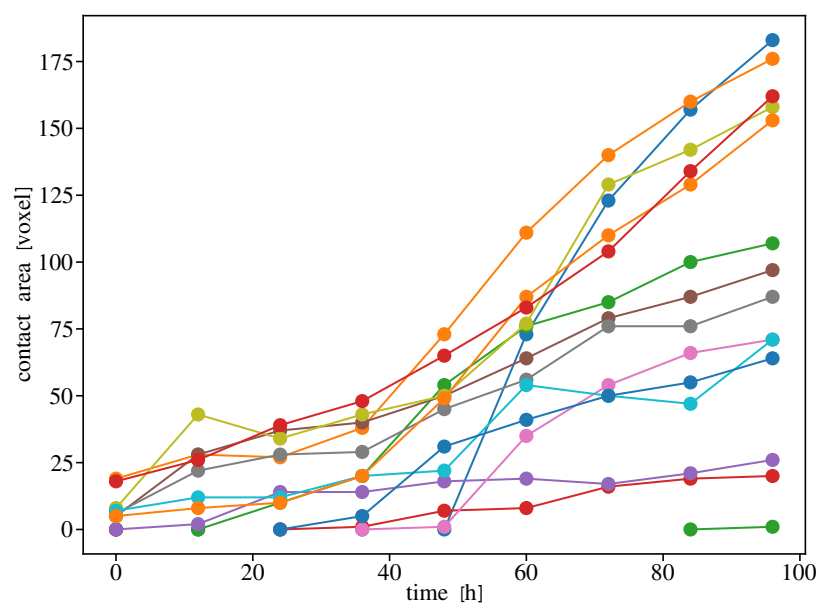

Figure 7. Time evolution of the contact areas of a grain with its neighbours. Not only the existing contacts tend to increase in size, but also new contacts generate during the exposition to high RH under constant load.

\section{Conclusions}

This study shows (and it does it quantitatively, i.e, through measurements) how the behaviour of water-sensitive materials is highly affected by the presence of water, even in its gaseous form. While exposing couscous to high relative humidity air, different processes are observed. The grains agglomerate (at the end of the test, they formed a semirigid cylinder), swell, lifting the vertical constant load for a considerable amount of time, but then lose resistance, causing an initial expansion followed by compression of the whole specimen. The decrease of the sample porosity is measured along the entire duration of the experiment and it is compared with the evolution in time of the specimen and average grain volumetric strains. Image correlation and Discrete Digital Image Correlation, allow to measure the deformations and kinematics at both macro and grain level (it is possible to follow each one of the 2200 complexly shaped grains through the whole experiment). To describe in detail and accurately the material behaviour, it is important to distinguish between these two scales of analysis, since the processes occurring at the micro level influence the overall macro response.

Finally, a simple exploration to the evolution of contacts is carried on. The contact areas generally increase, suggesting that this process might be highly connected with the agglomeration and so the exhibition of cohesion of the granular packing.

The analysis of the areas of contact could be investigated in more detail in the future. The strain of the surface of contacts could be measured and it could be correlated with the cementation/agglomeration of the grains.

Furthermore, the spatial distribution of water content together with the temporal evolution of the granular skeleton should be studied.

This project has received funding from the European Union's Horizon 2020 research and innovation program under the Marie Skłodowska-Curie grant agreement No. 812638 (CALIPER).

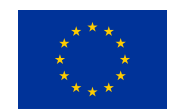

\section{References}

[1] D.G. Fredlund, H. Rahardjo, Soil mechanics for unsaturated soils (John Wiley \& Sons, 1993)

[2] J. Aguilera, J. del Valle, M. Karel, Trends Food Sci. Tech. 6, 149 (1995)

[3] G. Lumay, K. Traina, F. Boschini, V. Delaval, A. Rescaglio, R. Cloots, N. Vandewalle, J. Drug Deliv. Sci. Tec. 35, 207 (2016)

[4] M. Amin, M. Hossain, K. Roy, J. Food Eng. 65, 83 (2004)

[5] T. Morita, R.P. Singh, T. ASAE 22, 630 (1979)

[6] I. Siddiqui, S. Yiu, J. Jones, M. Kalab, Food Struct. 5, 17 (1986)

[7] T. Sasaki, J. Matsuki, Cereal Chem. 75, 525 (1998)

[8] G. Kamst, C. Bonazzi, J. Vasseur, J. Bimbenet, T. ASAE 45, 145 (2002)

[9] W. Cao, Y. Nishiyama, S. Koide, Int. J. Food Sci. Tech. 39, 899 (2004)

[10] A.D. Sharma, O.R. Kunze, T. ASAE 25, 465 (1982)

[11] S. Radl, E. Kalvoda, B.J. Glasser, J.G. Khinast, Powder Technol. 200, 171 (2010)

[12] L. Greenspan, J. Res. Natl. Bur. Stand. 81, 89 (1977)

[13] S.A. Hall, M. Bornert, J. Desrues, Y. Pannier, N. Lenoir, G. Viggiani, P. Bésuelle, Géotechnique 60, 315 (2010)

[14] O. Stamati, E. Andò, E. Roubin, R. Cailletaud, M. Wiebicke, G. Pinzon, C. Couture, R.C. Hurley, R. Caulk, D. Caillerie et al., J. Open Source Softw. 5, 2286 (2020) 